\title{
Leakage-Resilient Signatures with Graceful Degradation
}

\author{
Jesper Buus Nielsen ${ }^{1}$, Daniele Venturi ${ }^{2, \star}$, and Angela Zottarel ${ }^{1}$ \\ 1 Aarhus University \\ 2 Sapienza University of Rome
}

\begin{abstract}
We investigate new models and constructions which allow leakage-resilient signatures secure against existential forgeries, where the signature is much shorter than the leakage bound. Current models of leakage-resilient signatures against existential forgeries demand that the adversary cannot produce a new valid message/signature pair $(m, \sigma)$ even after receiving some $\lambda$ bits of leakage on the signing key. If $|\sigma| \leq \lambda$, then the adversary can just choose to leak a valid signature $\sigma$, and hence signatures must be larger than the allowed leakage, which is impractical as the goal often is to have large signing keys to allow a lot of leakage.

We propose a new notion of leakage-resilient signatures against existential forgeries where we demand that the adversary cannot produce $n=$ $\lfloor\lambda /|\sigma|\rfloor+1$ distinct valid message/signature pairs $\left(m_{1}, \sigma_{1}\right), \ldots,\left(m_{n}, \sigma_{n}\right)$ after receiving $\lambda$ bits of leakage. If $\lambda=0$, this is the usual notion of existential unforgeability. If $1<\lambda<|\sigma|$, this is essentially the usual notion of existential unforgeability in the presence of leakage. In addition, for $\lambda \geq|\sigma|$ our new notion still guarantees the best possible, namely that the adversary cannot produce more forgeries than he could have leaked, hence graceful degradation.

Besides the game-based notion hinted above, we also consider a variant which is more simulation-based, in that it asks that from the leakage a simulator can "extract" a set of $n-1$ messages (to be thought of as the messages corresponding to the leaked signatures), and no adversary can produce forgeries not in this small set. The game-based notion is easier to prove for a concrete instantiation of a signature scheme. The simulation-based notion is easier to use, when leakage-resilient signatures are used as components in larger protocols.

We prove that the two notion are equivalent and present a generic construction of signature schemes meeting our new notion and a concrete instantiation under fairly standard assumptions. We further give an application, to leakage-resilient identification.
\end{abstract}

\section{Introduction}

The problem of message authentication is one of the most basic in cryptography. Alice wants to transmit a message $m$ to Bob via an insecure channel, with the

* Part of the work done while at Aarhus University supported by the DFF Starting Grant 10-081612. 
guarantee that the message will reach the destination without any modification by a third party on the communication channel. In a world where public-key cryptography exists the latter can be achieved via a digital signature: Before sending $m$, Alice computes a signature $\sigma$ (via her signing key $s k$ ) of the message, and transmits $(m, \sigma)$ over the channel. The idea is that Bob can later verify the signature using Alice's verification key $v k$, and thus establish whether the received message is consistent with the original.

Traditionally, security of signatures schemes (and other primitives) is modeled in a black-box fashion where an adversary can only access the algorithms underlying the scheme as a black-box. For instance, in the case of a signature scheme, we require that no computationally bounded adversary is able to forge a signature of a message (with respect to some verification key $v k$ ) even given black-box access to an oracle returning signatures of arbitrarily chosen messages (computed via the signing key corresponding to $v k$ ) 1 However, as pointed out by recent research, the model above might be too restrictive, in that in practice there are several ways by which an adversary can learn partial information (a.k.a. leakage) on the secrets used within a cryptographic primitive, and thus easily step out of the security model. This includes so-called side-channel attacks, based on timings [27, power analysis [28] and electromagnetic radiation [35.

A large body of work has extended standard cryptographic definitions such that they can capture different flavours of security against leakage, both in the game-based setting (e.g. [1333130 24,14, 10,116]) and in the simulationbased setting 17/214/31. In the case of a signature scheme, a simple extension of the black-box setting requires that no computationally bounded adversary is able to forge a signature of a message (with respect to some verification key $v k$ ) even given black-box access to an oracle returning signatures on arbitrarily chosen messages (computed via the signing key corresponding to $v k$ ) and to a leakage oracle returning bounded (but otherwise arbitrary) information on the signing key $s k$. This is often referred to as the bounded leakage model, and on this we focus our work. See Section 1.2 for a discussion on other models.

The modeling above requires two necessary limitations. The first limitation is that the total amount of leakage must be smaller than the length of the signing key, as otherwise the entire key can be learned by the adversary, leaving no hope for security. The second limitation is that a signature has to be longer than the leakage bound, as otherwise a leakage query can just leak a forgery which is a valid attack against the security definition. A similar issue was already observed by Alwen, Dodis and Wichs [1], in their work on leakage-resilient public-key cryptography in the so-called bounded retrieval model. In this setting, the secret key is made intentionally large (say, 100 gigabytes) such that it may be infeasible/impractical for the attacker to download "too much" data (say, more than 1 gigabytes). Still, the length of the public key and the computational overhead are essentially independent from the size of the secret key. For the very same reason pointed out above, no signature scheme can be proven existentially unforgeable

${ }^{1}$ The restriction is of course that the forgery should not correspond to one of the messages asked to the oracle. 
in the bounded retrieval model, as the leakage could simply consist of a forgery. To tackle this issue the authors in [1] considered a weaker notion, which they name entropic unforgeability, where, after the leakage phase, the adversary is required to forge the signature of a message sampled from a (potentially adversarially chosen) distribution of high enough min-entropy (given the entire view of the adversary). [1] then shows that entropic unforgeability can be achieved in the random oracle model [3], by applying the Fiat-Shamir transform [16] to a certain class of interactive protocols.

In this work we propose more granular ways to model (bounded) leakage resilience for signature schemes where the length of the signature is smaller than the length of the secret key. In a nutshell, our simplest notion says that an adversary leaking $\lambda$ bits will always be able to produce $\lfloor\lambda /|\sigma|\rfloor$ forgeries, but not more than that. At first glance it may seem that our notion gives a weaker guarantee. However, the number of forgeries the adversary is required to produce strictly depends on the actual leakage, so if an adversary asks for no leakage (i.e. we are in the black-box model), our notion is equivalent to standard existential unforgeability, as now $\lfloor\lambda /|\sigma|\rfloor=0$. On the other hand, when leakage does happen, our definition offers a graceful degradation of security and, as we argue in more details below, still allows for interesting, non-trivial, applications.

\subsection{Our Contribution}

We investigate new models and constructions which allow leakage-resilient signatures secure against existential forgeries, where the signature is much shorter than the leakage bound. Our main contributions are discussed in detail below.

One-more unforgeability. As a first contribution, we state a variant of leakage resilience for signature schemes where the length of the secret key is much larger than the length of a signature 2 We name our notion one-more unforgeability, since it has a similar flavour to the unforgeability notion for blind signatures [34. The attacker (given the verification key $v k$ ) can access a signing oracle and a leakage oracle; at the end he has to output $n$ forgeries $\left(m_{1}, \sigma_{1}\right), \ldots,\left(m_{n}, \sigma_{n}\right)$ and wins the game if and only if all the forgeries are valid, the messages are pairwise distinct, and $n$ is strictly larger than the number of forgeries one could have leaked via leakage queries. See Section 3 for a precise definition.

We also formulate a seemingly stronger variant, which we name constrainedone-more unforgeability. Here we introduce a simulator $S$ which first looks at the state of the adversary $A$ after the leakage phase ended and then defines a set of messages $\mathcal{Q}^{*}$ of size strictly smaller than $n$, as defined above. A signature scheme is secure in this setting if, for all $A$, there exists such a simulator for which $A$ is not able to forge a message which is not contained in $\mathcal{Q}^{*}$ (and was not already asked to the signing oracle). This captures the intuition that the forgeries are

\footnotetext{
${ }^{2}$ Note that this in general encompasses schemes with short signatures, and not necessarily signature schemes in the bounded retrieval model.
} 
already fixed after the leakage is ended, and the adversary is "constrained" in the sense that those are the only messages for which he can forge 3

We show that one-more unforgeability and constrained-one-more unforgeability are equivalent. The tricky direction is to show that the former implies the latter. The intuition is using an adversary breaking constrained-one-more unforgeability and rewinding him to obtain a sufficiently large set of forgeries: if at each rewinding we use a strictly larger set $\mathcal{Q}^{*}$ (including all previous forgeries output by the adversary), after $n$ steps we end-up with $n$ forgeries which allow to break one-more unforgeability. The actual analysis is more involved, as we need to take care of the fact that we are rewinding the adversary at the point where he is already committed to the leakage.

A construction. As a second contribution we present a scheme achieving onemore-unforgeability, based on a perfectly hiding (homomorphic) commitment scheme and a non-interactive zero knowledge argument of knowledge system.

The secret key consists of the coefficients $\delta_{i}$ of a $d$-degree polynomial $\delta(\cdot)$ over a finite field, together with the openings $r_{i}$ for the commitments $\operatorname{com}_{i}$ to $\delta_{i}$. The verification key consists of the set of all $\operatorname{com}_{i}$ together with a common reference string for the argument system. To sign a message $m$, we compute $\delta(m)$ and we produce a zero-knowledge argument of knowledge that the evaluation of the polynomial was performed correctly using the coefficients whose commitments are in the verification key. The signature consists of such an argument.

We prove that the scheme is one-more unforgeable whenever the commitment is perfectly hiding (and computationally binding), as long as the leakage is smaller than $(1 / 2-o(1)) \cdot|s k|$. We also show a particular instantiation, using standard building blocks such as Pedersen commitments 32 and Groth-Sahai proofs [1920]. Security follows from the DLIN assumption [5]. We remark that for our concrete instantiation it is indeed the case that the length of a signature is essentially independent of the length of the secret key.

Application to identification protocols. Besides being a notion of theoretical interest, we also show that one-more unforgeability can be applied in the context of identification protocols. We focus on the public-key setting, where a prover $P$ wants to be identified from a verifier $\mathrm{V}$ holding $\mathrm{P}$ 's public key.

Following [1, we define security in the presence of leakage by considering an adversary having black-box access to the prover and to a leakage oracle

\footnotetext{
${ }^{3}$ We note that constrained-one-more unforgeability is strictly stronger than entropic unforgeability [1]. If a scheme is constrained-one-more unforgeable, then after the leakage is done, a poly-sized set of messages $\mathcal{Q}^{*}$ is defined and the adversary cannot forge for a message outside $\mathcal{Q}^{*}$, whereas a high entropy message will hit inside $\mathcal{Q}^{*}$ with negligible probability. On the other hand consider a signature scheme where a signature is given as $\sigma=\Pi^{-1}(m)$ for a one-way trapdoor permutation $\Pi$ hard to invert on high-entropy $m$. Such a scheme is entropic secure in the presence of $\lambda=0$ bits of leakage, by definition, but is clearly not constrained-one-more unforgeable in the presence of $\lambda=0$ bits of leakage, as the adversary can always sample one more random message/signature pair as $m=\Pi(\sigma)$ for random $\sigma$.
} 
(depending on the prover's secret key) in a first phase. In a second phase the adversary is given one chance to convince the verifier. The above notion is reminiscent of so-called active security 2325 .

We show that the classical protocol for public-key identification, where the verifier challenges the prover with a random message and the prover has to respond with a signature on that message, achieves the above notion of active security with leakage, provided that the underlying signature scheme is constrained-one-more unforgeable.

\subsection{Other Related Work}

In this work (similarly to [1]0[15]) we focus on bounded leakage resilience, i.e., we assume that there is an a-priori upper bound on the length of the maximum tolerated leakage. Furthermore, we consider a setting where the leakage can only depend on the signing key and not on the full state of the signer (including, e.g., the signer's random coins). A strictly stronger notion of fully leakage-resilient signatures (where the leakage is bounded but can depend on the entire state of the signer) was considered in [246].

In the continual leakage setting $7 \longdiv { 9 } 2 9 6$, there is no a priori bound on the length of the leakage. This requires an efficient procedure to update the secret key (while leaving the public key unchanged), and to assume that the leakage is bounded only between two updates (and during the update process itself).

An independent line of research (see, e.g. [22 26]) aims at constructing signature schemes (in the black-box model) which are as short as possible. Even though this is not our purpose, we believe that our notions could have interesting implications in this setting, when studying leakage resilience of such schemes.

\section{Preliminaries}

\subsection{Notation}

For $a, b \in \mathbb{R}$, we let $[a, b]=\{x \in \mathbb{R} ; a \leq x \leq b\} ;$ for $a \in \mathbb{N}$ we let $[a]=$ $\{1,2, \ldots, a\}$. If $x$ is a string, we denote its length by $|x|$; if $\mathcal{X}$ is a set, $|\mathcal{X}|$ represents the number of elements in $\mathcal{X}$. When $x$ is chosen randomly in $\mathcal{X}$, we write $x \leftarrow \mathcal{X}$. When $\mathrm{A}$ is an algorithm, we write $y \leftarrow \mathrm{A}(x)$ to denote a run of $\mathrm{A}$ on input $x$ and output $y$; if $\mathrm{A}$ is randomized, then $y$ is a random variable and $\mathrm{A}(x ; r)$ denotes a run of $\mathrm{A}$ on input $x$ and randomness $r$. An algorithm $\mathrm{A}$ is probabilistic polynomial-time (PPT) if $\mathrm{A}$ is randomized and for any input $x, r \in\{0,1\}^{*}$ the computation of $\mathrm{A}(x ; r)$ terminates in at most poly $(|x|)$ steps.

Throughout the paper we let $\kappa$ denote the security parameter. We say that a function $\nu: \mathbb{N} \rightarrow \mathbb{R}$ is negligible in the security parameter $\kappa$ if $\nu(\kappa)=\kappa^{-\omega(1)}$. For two ensembles $\mathcal{X}=\left\{X_{\kappa}\right\}_{\kappa \in \mathbb{N}}$ and $\mathcal{Y}=\left\{Y_{\kappa}\right\}_{\kappa \in \mathbb{N}}$, we write $\mathcal{X} \equiv \mathcal{Y}$ if they

\footnotetext{
${ }^{4}$ In fact, as argued in [2], without leakage the signature based protocol is even secure against man-in-the-middle attacks. It is not hard to see, however, that our result does not extend to man-in-the-middle security.
} 
are identically distributed and $\mathcal{X} \approx_{s} \mathcal{Y}$ to denote that the statistical distance between the two distributions is negligible in the security parameter. We say that $\mathcal{X}$ and $\mathcal{Y}$ are computationally indistinguishable if for all PPT distinguishers $\mathrm{D}$ it holds that $\left|\mathbb{P}\left[\mathrm{D}\left(1^{\kappa}, X\right)=1\right]-\mathbb{P}\left[\mathrm{D}\left(1^{\kappa}, Y\right)=1\right]\right|$ is negligible in $\kappa$.

The min-entropy of a random variable $X$ over a set $\mathcal{X}$ is defined as $\mathbb{H}_{\infty}(X):=$ $-\log \max _{x} \mathbb{P}[X=x]$ and represents the best chance of guessing $X$ by an unbounded adversary. Average min-entropy captures how hard it is to guess $X$ on average, given some side information $Z$ (possibly related to $X$ ):

$$
\widetilde{\mathbb{H}}_{\infty}(X \mid Z)=-\log \mathbb{E}_{z}\left[\max _{x} \mathbb{P}[X=x \mid Z=z]\right] .
$$

The min-entropy of a distribution conditioned to some side information cannot decrease more than the bit-length of the side information itself:

Lemma 1 ([12]). For all random variables $X \in \mathcal{X}$ and $\Lambda \in\{0,1\}^{\lambda}$ we have that $\widetilde{\mathbb{H}}_{\infty}(X \mid \Lambda) \geq \mathbb{H}_{\infty}(X)-\lambda$.

We let $\mathcal{O}^{\ell}(s)$ be an oracle parametrized by a value $s$, which takes as input efficiently computable functions $f:\{0,1\}^{*} \rightarrow\{0,1\}^{*}$ and outputs $f(s)$, returning a total of at most $\ell$ bits.

\subsection{Commitment Schemes}

A (non-interactive) commitment scheme $\mathcal{C O} \mathcal{M}$ is a tuple of algorithms (Setup, Commit), defined as follows: (1) Algorithm Setup takes as input the security parameter and outputs a public key $p k$; (2) Algorithm Commit takes as input a message $m \in \mathcal{M}$, randomness $r \in \mathcal{R}$, the public key $p k$ and outputs a value com $\in \mathcal{C}$. To open a commitment com we output $(m, r)$; an opening is valid if and only if $\mathrm{com}=\operatorname{Commit}(m ; r)$.

A commitment scheme has two properties, known as binding and hiding. In Section 4 we need a scheme with the following flavour.

Computationally Binding : For any PPT adversary A, the following is negligible:

$$
\mathbb{P}\left[\operatorname{Commit}\left(m_{0} ; r_{0}\right)=\operatorname{Commit}\left(m_{1} ; r_{1}\right): \begin{array}{c}
p k \leftarrow \operatorname{Setup}\left(1^{\kappa}\right) ; \\
\left(\left(m_{0}, r_{0}\right),\left(m_{1}, r_{1}\right)\right) \leftarrow \mathrm{A}(p k)
\end{array}\right] .
$$

Statistically Hiding : For all messages $m_{0}, m_{1} \in \mathcal{M}$, we have that

$$
\left\{p k, \operatorname{Commit}\left(p k, m_{0}\right)\right\}_{\kappa \in \mathbb{N}} \approx_{s}\left\{p k, \operatorname{Commit}\left(p k, m_{1}\right)\right\}_{\kappa \in \mathbb{N}},
$$

where the two ensembles are considered as random variables over the choice of the randomness to generate $p k \leftarrow \operatorname{Setup}\left(1^{\kappa}\right)$ and to compute the commitment. If the two ensembles are identically distributed, we say that the commitment is perfectly hiding. 
Whenever $\mathcal{M}$ and $\mathcal{R}$ are a finite field $\mathbb{F}$, we say that $\mathcal{C O} \mathcal{M}$ is linearly homomorphic in the following sense: Given commitments com and $\mathrm{com}^{\prime}$ and a field element $c \in \mathbb{F}$, one can compute commitments com $^{*}$ and $\mathrm{com}^{\prime \prime}$ such that being able to open com and $\mathrm{com}^{\prime}$ to $m$ and $\mathrm{m}^{\prime}$ (respectively) allows to open $\mathrm{com}^{*}$ to $m+m^{\prime}$ and $c o m^{\prime \prime}$ to $c \cdot m$. We will write the mapping $\left(c o m, c o m^{\prime}\right) \mapsto c o m^{*}$ as $\mathrm{com} \cdot \mathrm{com}^{\prime}$ and the mapping $(c, \mathrm{com}) \mapsto \mathrm{com}^{\prime \prime}$ as $\mathrm{com}^{c}$. Similarly, for the opening information we will write the mappings as $c o m^{*}=\operatorname{Commit}\left(p k, m+m^{\prime} ; r+r^{\prime}\right)$ and $c o m{ }^{\prime \prime}=\operatorname{Commit}(p k, c \cdot m ; c \cdot r)$. The above can be generalized to abstract operations over $\mathcal{M}, \mathcal{R}$ and $\mathcal{C}$, but for simplicity, and to be consistent with the concrete instantiation given in Section 4.2 we stick to this formulation here.

\subsection{Non-interactive Zero-Knowledge Arguments of Knowledge}

For a relation $\mathfrak{R} \subseteq\{0,1\}^{*} \times\{0,1\}^{*}$, the language associated with $\mathfrak{R}$ is $\mathfrak{L}_{\mathfrak{R}}=\{x$ : $\exists w$ s.t. $(x, w) \in \mathfrak{R}\}$. A non-interactive argument system $\mathcal{N} \mathcal{I} \mathcal{Z} \mathcal{K}$ for a relation $\mathfrak{R}$ is a tuple of algorithms (Init, Prove, Ver), defined as follows: (1) Algorithm Init takes as input the security parameter and outputs a common reference string crs $\leftarrow \operatorname{Init}\left(1^{\kappa}\right) ;(2)$ Algorithm Prove takes as input a pair $(x, w)$ such that $(x, w) \in$ $\mathfrak{R}$ and outputs an argument $\pi ;(3)$ Algorithm Ver takes as input a pair $(x, \pi)$ and outputs a judgement in $\{0,1\}$.

We require the following properties for $\mathcal{N} \mathcal{I} \mathcal{Z} \mathcal{K}$ [36 10].

Completeness: For every $(x, w) \in \mathfrak{R}$ we have that

$\operatorname{Pr}\left[\operatorname{Ver}(\operatorname{crs},(x, \pi))=1: \operatorname{crs} \leftarrow \operatorname{Init}\left(1^{\kappa}\right) ; \pi \leftarrow \operatorname{Prove}(\operatorname{crs},(x, w))\right] \geq 1-\operatorname{negl}(\kappa)$.

Multi-theorem zero-knowledge: There exists a PPT simulator $\operatorname{Sim}=\left(\operatorname{Sim}_{1}\right.$, $\left.\operatorname{Sim}_{2}\right)$ such that, for all PPT adversaries A, the ensembles $\{\operatorname{Real}(\kappa)\}_{\kappa \in \mathbb{N}}$ and $\{\operatorname{Simu}(\kappa)\}_{\kappa \in \mathbb{N}}$ are computationally close, where

$$
\begin{aligned}
\operatorname{Real}(\kappa) & :=\left\{\operatorname{crs} \leftarrow \operatorname{Init}\left(1^{\kappa}\right) ; \text { out } \leftarrow \mathrm{A}^{\operatorname{Prove}(\mathrm{crs}, \cdot)}(\mathrm{crs})\right\} \\
\operatorname{Simu}(\kappa) & :=\left\{(\mathrm{crs}, t k) \leftarrow \operatorname{Sim}_{1}\left(1^{\kappa}\right) ; \text { out } t^{\prime} \leftarrow \mathrm{A}^{\widetilde{\operatorname{Sim}_{2}}(t k, \cdot)}(\mathrm{crs})\right\}
\end{aligned}
$$

and $\widetilde{\operatorname{Sim}}_{2}(t k,(x, w))$ outputs $\operatorname{Sim}_{2}(t k, x)$ if $(x, w) \in \mathfrak{R}$, and $\perp$ otherwise.

Simulation extractability: There exists a PPT algorithm $\mathrm{Xtr}=\left(\mathrm{Xtr}_{1}, \mathrm{Xtr} 2\right)$ such that, for all PPT adversaries A, we have that

$$
\mathbb{P}\left[\begin{array}{c}
(\mathrm{crs}, t k, x k) \leftarrow \mathrm{Xtr}_{1}\left(1^{k}\right) ;(x, \pi) \leftarrow \mathrm{A}^{\operatorname{Sim}_{2}(t k, \cdot)}(\mathrm{crs}) \\
w \leftarrow \operatorname{Xtr}_{2}(x k,(x, \pi)) ;(x, w) \notin \mathfrak{R} \wedge(x, \pi) \notin \mathcal{Q} \wedge \operatorname{Ver}(\operatorname{crs},(x, \pi))=1
\end{array}\right]
$$

is negligible, where the list $\mathcal{Q}$ contains the successful pairs $\left(x_{i}, \pi_{i}\right)$ that $\mathrm{A}$ has queried to $\operatorname{Sim}_{2}$. We say that $\mathcal{N} \mathcal{I} \mathcal{Z} \mathcal{K}$ is true simulation-extractable if oracle $\operatorname{Sim}_{2}(t k, x)$ is replaced by $\widehat{\operatorname{Sim}_{2}}(t k,(x, w))$ that outputs the same as $\operatorname{Sim}_{2}(t k, x)$ if and only if $(x, w) \in \mathfrak{R}$ (and outputs $\perp$ otherwise). 


\section{One-More Unforgeability}

A signature scheme is a triple of algorithms $\mathcal{S S}=$ (KGen, Sign, Verify) defined as follows: (1) The key generation algorithm takes as input the security parameter $\kappa$ and outputs a verification key/signing key pair $(v k, s k) \leftarrow \mathrm{KGen}\left(1^{\kappa}\right)$; (2) The signing algorithm takes as input a message $m \in \mathcal{M}$ and the signing key $s k$ and outputs a signature $\sigma \leftarrow \operatorname{Sign}(s k, m)$; (3) The verification algorithm takes as input the verification key $v k$ and a pair $(m, \sigma)$ and outputs $\operatorname{Verify}(v k,(m, \sigma)) \in$ $\{0,1\}$. We denote by $|\sigma|$ the size of a $\operatorname{signature~output}$ via $\operatorname{Sign}(s k, \cdot)$.

Given a signature scheme $\mathcal{S} \mathcal{S}$, consider the following experiment $\operatorname{Exp}_{\mathcal{S} \mathcal{S}, \mathrm{A}}^{\text {one-me }}(\kappa$, $\ell, \gamma)$ running with a PPT adversary A and parametrized by the security parameter $\kappa \in \mathbb{N}$, the leakage bound $\ell \in \mathbb{N}$ and the slack parameter $\gamma \in(0,1]$ :

1. Compute $(v k, s k) \leftarrow \mathrm{KGen}\left(1^{\kappa}\right)$ and give $v k$ to $\mathrm{A}$.

2. The adversary A can adaptively access oracles $\operatorname{Sign}(s k, \cdot)$ and $\mathcal{O}^{\ell}(s k, \cdot)$, where $\mathcal{O}^{\ell}(s k, f)$ returns $f(s k)$. We let $\Lambda \in\{0,1\}^{\lambda}$ be the total information returned by $\mathcal{O}^{\ell}$ (with $\lambda \leq \ell$ ), and we write $\mathcal{Q}$ for the set of messages $A$ forwarded to the signing oracle.

3. A outputs $n$ pairs $\left(m_{1}, \sigma_{1}\right), \ldots,\left(m_{n}, \sigma_{n}\right)$.

4. The experiment outputs 1 iff if the following conditions are satisfied:

(a) $\operatorname{Verify}\left(v k,\left(m_{i}, \sigma_{i}\right)\right)=1$ and $m_{i} \notin \mathcal{Q}$, for all $i \in[n]$.

(b) The messages $m_{1}, \ldots, m_{n}$ are pairwise distinct.

(c) $n \geq\lfloor\lambda /(\gamma|\sigma|)\rfloor+1$.

Definition 1 (One-more unforgeability). We say that $\mathcal{S S}=(\mathrm{KGen}$, Sign, Verify) is $(\ell, \gamma, \varepsilon)$-one-more unforgeable if for every PPT adversary $\mathrm{A}$ we have that $\mathbb{P}\left[\operatorname{Exp}_{\mathcal{S} \mathcal{S}, \mathrm{A}}^{\text {one-more }}(\kappa, \ell, \gamma)=1\right] \leq \varepsilon$. Whenever $\varepsilon$ is negligible in the security parameter, we simply say that $\mathcal{S} \mathcal{S}$ is $(\ell, \gamma)$-one-more unforgeable.

Remark 1 (on $\gamma$ ). The parameter $\gamma$ specifies how close to optimal security $\mathcal{S} \mathcal{S}$ is. In particular, in case $\gamma=1$ one-more unforgeability requires that A cannot forge even a single signature more than what it could have leaked via leakage queries. As $\gamma$ decreases, so does the strength of the signature scheme (the extreme case being $\gamma=|\mathcal{M}|^{-1}$, where we have no security).

Note that the number of signatures the adversary has to forge depends on the length of the leakage he asks to see. In particular $(\ell, \gamma)$-one-more unforgeability implies standard unforgeability for any adversary asking no leakage $(\lambda=0)$.

Finally, we remark that for any $\gamma \in(0,1]$ we have that $(\ell, \gamma)$-one-more unforgeability implies $\left(\ell^{\prime}, \gamma\right)$-one-more unforgeability for all $\ell^{\prime} \leq \ell$.

\subsection{An Alternative Definition}

Definition 1 may seem a weak security guarantee for a signature scheme, as an adversary is able to forge a certain number of signatures. If the messages to forge could be chosen at will at any time, this would be a rather useless security guarantee. Here, we state a seemingly stronger flavour of one-more unforgeability 
where a simulator can look at the state of the adversary after he is done with leakage queries and output a set $\mathcal{Q}^{*} \subset \mathcal{M}$, of size less than $n$, thought of as the messages corresponding to the forgeries leaked so far; now the adversary is successful if he can produce a forgery for a message of his choice not contained in $\mathcal{Q}^{*}$ (and not already asked to the signing oracle). In a certain sense, we get a notion that is similar to the standard unforgeability notion, with the twist that the adversary can ask a few extra signing queries (via leakage queries, though).

Given a signature scheme $\mathcal{S S}$, consider the experiment $\operatorname{Exp}_{\mathcal{S} \mathcal{S}, \mathrm{A}, \mathcal{S}}^{\text {poly-one-more }}(\kappa$, $\ell, \gamma)$ below, running with a PPT adversary $A=\left(A_{1}, A_{2}\right)$ and a PPT simulator $S$ and parametrized by the security parameter $\kappa \in \mathbb{N}$, the leakage bound $\ell \in \mathbb{N}$ and the slack parameter $\gamma \in(0,1]$ :

1. Compute $(v k, s k) \leftarrow \mathrm{KGen}\left(1^{\kappa}\right)$ and give $v k$ to $\mathrm{A}$.

2. The adversary $A_{1}$ can adaptively access oracles $\operatorname{Sign}(s k, \cdot)$ and $\mathcal{O}^{\ell}(s k, \cdot)$, where $\mathcal{O}^{\ell}(s k, f)$ returns $f(s k)$. We let $\Lambda \in\{0,1\}^{\lambda}$ be the total information returned by $\mathcal{O}^{\ell}$ (with $\lambda \leq \ell$ ), and we write $\mathcal{Q}$ for the set of messages $\mathrm{A}_{1}$ forwarded to the signing oracle.

3. Let $s t$ be the state of $\mathrm{A}_{1}$ at the end of step 2 above, i.e., all his inputs, all his random choices, and all replies from the oracles. The simulator is given $s t$ and outputs $\mathcal{Q}^{*} \leftarrow \mathrm{S}\left(1^{\kappa}, v k, s t\right)$ such that $\mathcal{Q}^{*} \subset \mathcal{M}$ and $\left|\mathcal{Q}^{*}\right| \leq\lfloor\lambda /(\gamma|\sigma|)\rfloor$.

4. $\mathrm{A}_{2}$ is given $\mathcal{Q}^{*}$ and $s t$ and outputs a forgery $\left(m^{*}, \sigma^{*}\right)$.

5. The experiment outputs 1 iff $\operatorname{Verify}\left(v k,\left(m^{*}, \sigma^{*}\right)\right)=1$ and $m^{*} \notin \mathcal{Q} \cup \mathcal{Q}^{*}$.

Definition 2 (Poly-constrained one-more unforgeability). We say that $\mathcal{S S}=($ KGen, Sign, Verify) is $(\ell, \gamma, \varepsilon)$-poly-constrained one-more unforgeable if for every PPT adversary $\mathrm{A}$ there exists a PPT simulator $\mathrm{S}$ such that

$$
\mathbb{P}\left[\operatorname{Exp}_{\mathcal{S} \mathcal{S}, \mathrm{A}, \mathrm{S}}^{\text {poly-sim-one-more }}(\kappa, \ell, \gamma)=1\right] \leq \varepsilon .
$$

Whenever $\varepsilon$ is negligible in the security parameter, we simply say that $\mathcal{S} \mathcal{S}$ is $(\ell, \gamma)$-poly-constrained one-more unforgeable.

\subsection{Yet another Alternative Definition}

Definition 2 requires that $\mathcal{Q}^{*}$ can be computed in poly-time, effectively requiring that the adversary knows the small set of forgeries he leaked. In most applications we are aware of, it seems, however, enough that such a small set exists. And, there seems to be a difference between these notions. Consider an adversary who leaks a few values of the form $v_{i}=H\left(m_{i}\right) \oplus \sigma_{i}$, where $H$ is a hash function, for random messages $m_{i}$ (with $i \in[n]$ ) and $\sigma_{i}$ a signature on $m_{i}$. Given any $m_{i}$ as input it can compute a "forgery" $\sigma_{i}=v_{i} \oplus H\left(m_{i}\right)$, but until it is given $m_{i}$ it does not know the set of messages it can forge signatures on, at least it would be hard to compute this set efficiently in a black-box manner. We formulate a security notion which still considers leakage of a few such "unknown" $\sigma_{i}$ as benign.

We simply restate Definition 2 , but we now allow $S$ unbounded computing time. We can massage this relaxed definition a bit to get a simpler, equivalent definition. Consider the following generic simulator $S_{\min }\left(1^{\kappa}, v k, s t\right)$ : it iterates 
over all $\mathcal{Q}^{*} \subset \mathcal{M}$ with $\left|\mathcal{Q}^{*}\right| \leq\lfloor\lambda /(\gamma|\sigma|)\rfloor$ and computes the probability $p_{\mathcal{Q}^{*}}$ that $\mathrm{A}_{2}\left(\mathcal{Q}^{*}, s t\right)$ outputs $\left(m^{*}, \sigma^{*}\right)$ such that $\operatorname{Verify}\left(v k,\left(m^{*}, \sigma^{*}\right)\right)=1$ and $m^{*} \notin \mathcal{Q} \cup \mathcal{Q}^{*}$. It then outputs the $\mathcal{Q}^{*}$ minimizing $p_{\mathcal{Q}^{*}}$. It is clear that if for some adversary A there exists an unbounded simulator $\mathrm{S}$ fulfilling Definition 2 for $\mathrm{A}$, then also $S_{\min }$ will fulfil Definition 2 for $A$. Hence we can equivalently hardwire $S_{\min }$ into the definition. If we at the same time use that the expected value of a random value over $\{0,1\}$ is equal to the probability that it is 1 , we get the below more compact definition. Consider the following experiment $\operatorname{Exp}_{\mathcal{S} \mathcal{S}, \mathrm{A}}^{\mathrm{sim}-\text { one-more }}(\kappa, \ell, \gamma)$ :

1. Compute $(v k, s k) \leftarrow \mathrm{KGen}\left(1^{\kappa}\right)$ and give $v k$ to $\mathrm{A}_{1}$.

2. The adversary $\mathrm{A}_{1}$ can adaptively access oracles $\operatorname{Sign}(s k, \cdot)$ and $\mathcal{O}^{\ell}(s k, \cdot)$, where $\mathcal{O}^{\ell}(s k, f)$ returns $f(s k)$. We let $\Lambda \in\{0,1\}^{\lambda}$ be the total information returned by $\mathcal{O}^{\ell}$ (with $\lambda \leq \ell$ ), and we write $\mathcal{Q}$ for the set of messages $\mathrm{A}_{1}$ forwarded to the signing oracle.

3. Let $s t$ be the state of $\mathrm{A}_{1}$ at the end of step 2 above.

4. Output

$$
\min _{\substack{\mathcal{Q}^{*} \subset \mathcal{M}: \\\left|\mathcal{Q}^{*}\right| \leq\lfloor\lambda /(\gamma|\sigma|)\rfloor}}\left(\mathbb{P}\left[\left(m^{*}, \sigma^{*}\right) \leftarrow \mathrm{A}_{2}\left(\mathcal{Q}^{*}, s t\right): \operatorname{Verify}\left(v k,\left(m^{*}, \sigma^{*}\right)\right) \wedge m^{*} \notin \mathcal{Q} \cup \mathcal{Q}^{*}\right]\right) .
$$

Definition 3 (Constrained one-more unforgeability). We say that $\mathcal{S} \mathcal{S}=$ (KGen, Sign, Verify) is $(\ell, \gamma, \varepsilon)$-constrained one-more unforgeable if it holds that $\mathbb{E}\left[\operatorname{Exp}_{\mathcal{S S}, \mathrm{A}}^{\mathrm{sim}-\text { one-more }}(\kappa, \ell, \gamma)\right] \leq \varepsilon$ for every $P P T$ adversary $\mathrm{A}$, where the expected value is over the random choices used to generate $(v k, s k)$ and the random choices of $\mathrm{A}_{1}$. Whenever $\varepsilon$ is negligible in the security parameter, we simply say that $\mathcal{S} \mathcal{S}$ is $(\ell, \gamma)$-constrained one-more unforgeable.

\subsection{Equivalence of Two Definitions}

We argue below that one-more unforgeability and constrained-one-more unforgeability are equivalent. It is clear that security under Definition 2 implies security under Definition 3 We conjecture that Definition 3 is strictly weaker than Definition 2 .

Theorem 1. Definition 1 and Definition $[$ are equivalent up to a constant factor 4 in security.

Proof. For space reasons, we prove only that Definition 1 implies Definition 3 . the proof of the other direction can be found in the full version. We give a proof by contradiction. Assume there exists a polynomial $\varepsilon$ and PPT adversary $\mathrm{A}^{\prime}=\left(\mathrm{A}_{1}^{\prime}, \mathrm{A}_{2}^{\prime}\right)$ such that $\mathbb{E}\left[\operatorname{Exp}_{\mathcal{S} \mathcal{S}, \mathrm{A}^{\prime}}^{\text {sim-ore-more }}(\kappa, \ell, \gamma)\right]>\varepsilon$ for infinitely many values of $\kappa$.

Since $0 \leq \mathbb{E}\left[\operatorname{Exp}_{\mathcal{S} \mathcal{S} \text {. } \mathrm{A}^{\prime}}^{\text {sime-more }}(\kappa, \ell, \gamma)\right] \leq 1$ this implies that $\mathbb{P}\left[\operatorname{Exp}_{\mathcal{S} \mathcal{S}, \mathrm{A}^{\prime}}^{\text {sim-one-more }}(\kappa\right.$, $\ell, \gamma) \geq \varepsilon / 2] \geq \varepsilon / 2$ for infinitely many values of $\kappa$. Let $E$ be the event that $\mathbb{P}\left[\operatorname{Exp}_{\mathcal{S} \mathcal{S}, A^{\prime}}^{\text {sime-more }}(\kappa, \ell, \gamma) \geq \varepsilon / 2\right]$.

We now describe $A$, running in experiment $\operatorname{Exp}_{\mathcal{S} \mathcal{S}, \mathrm{A}}^{\text {one-me }}(\kappa, \ell, \gamma)$. When reading the description keep in mind that it is defined to work when $E$ occurs. 
1. Receive the verification key $v k$ and initialize $\mathcal{Q}^{*}=\emptyset$.

2. Run $\mathrm{A}_{1}^{\prime}\left(1^{\kappa}, v k\right)$ and simulate leakage queries and signature queries using oracles $\mathcal{O}^{\ell}(s k, \cdot)$ and $\operatorname{Sign}(s k, \cdot)$. Let $\Lambda \in\{0,1\}^{\lambda}$ be the overall information retrieved by $A_{1}^{\prime}$.

3. Define $n:=\lfloor\lambda /(\gamma|\sigma|)\rfloor+1$. Repeat the following steps, for $i=1, \ldots, n$ :

(a) Run $8\left(\log _{2}(n)+\kappa\right) / \varepsilon$ copies of $\mathrm{A}_{2}^{\prime}\left(1^{\kappa}, s t, \mathcal{Q}^{*}\right)$ in parallel. If any of the copies outputs $\left(m_{i}^{*}, \sigma_{i}^{*}\right)$ such that $\operatorname{Verify}\left(v k,\left(m^{*}, \sigma^{*}\right)\right)=1$ and $m^{*} \notin$ $\mathcal{Q} \cup \mathcal{Q}^{*}$, then go to the next step, otherwise give up and terminate.

(b) Set $\mathcal{Q}^{*}:=\mathcal{Q}^{*} \cup\left\{m_{i}^{*}\right\}$ for one of the forgeries from above.

4. Output $\left(m_{1}^{*}, \sigma_{1}^{*}\right), \ldots,\left(m_{n}^{*}, \sigma_{n}^{*}\right)$.

Assume that $E$ occurs. Then the probability that any copy $\mathrm{A}_{2}^{\prime}\left(1^{\kappa}, s t, \mathcal{Q}^{*}\right)$ in Step 1 outputs $\left(m_{i}^{*}, \sigma_{i}^{*}\right)$ such that $\operatorname{Verify}\left(v k,\left(m^{*}, \sigma^{*}\right)\right)=1$ and $m^{*} \notin \mathcal{Q} \cup \mathcal{Q}^{*}$ is $\geq \varepsilon / 2$. Hence one of the copies will output such $\left(m_{i}^{*}, \sigma_{i}^{*}\right)$, except with probability $2^{-\log _{2}(n)-\kappa}$, by construction. Thus, by a union bound, the probability that $\mathrm{A}$ gives up in any of the iterations is at most $n \cdot 2^{-\log _{2}(n)-\kappa}=2^{-\kappa}$.

Clearly, when $A$ does not give up in any of the iterations, we have that $\operatorname{Exp}_{\mathcal{S} \mathcal{S}, \mathrm{A}}^{\text {one }}(\kappa, \ell, \gamma)=1$. Hence $\mathbb{P}\left[\operatorname{Exp}_{\mathcal{S} \mathcal{S}, \mathrm{A}}^{\text {one-more }}(\kappa, \ell, \gamma)=1\right] \geq \mathbb{P}[E]\left(1-2^{-\kappa}\right)=$ $\varepsilon\left(1-2^{-\kappa}\right) / 2>\varepsilon / 4$ for infinitely many values of $\kappa$. This concludes the proof as $\mathrm{A}$ is PPT.

\section{Construction}

We give a construction of a one-more unforgeable signature scheme (cf. Definition 1) based on the following building blocks:

- A non-interactive zero knowledge argument of knowledge system $\mathcal{N} \mathcal{I} \mathcal{Z} K=$ (Init, Prove, Ver).

- A perfectly hiding and computationally binding, linearly homomorphid5 commitment scheme $\mathcal{C O} \mathcal{M}=$ (Setup, Commit), with message and randomness space equal to a finite field $\mathbb{F}$.

Our scheme $\mathcal{S S}=($ KGen, Sign, Verify) has message space equal to $\mathbb{F}$ and is described below:

Key Generation. Run $p k \leftarrow \operatorname{Setup}\left(1^{\kappa}\right)$ and crs $\leftarrow \operatorname{Init}\left(1^{\kappa}\right)$. For some parameter $d \in \mathbb{N}$, sample $\delta_{0}, \ldots, \delta_{d}$ and $r_{0}, \ldots, r_{d}$ uniformly from $\mathbb{F}$, and compute commitments $\operatorname{com}_{i}=\operatorname{Commit}\left(p k, \delta_{i} ; r_{i}\right)$ for $i=0, \ldots, d$. Let $\boldsymbol{\delta}=\left(\delta_{0}, \ldots, \delta_{d}\right)$ and $\mathbf{r}=\left(r_{0}, \ldots, r_{d}\right)$; output $s k=(\boldsymbol{\delta}, \mathbf{r})$ and $v k=\left(\mathrm{crs}, p k,\left\{\operatorname{com}_{i}\right\}_{i=0}^{d}\right)$.

Signature. To sign a message $m \in \mathbb{F}$, let $\delta(X)$ be the degree $d$ polynomial having $\delta_{i}$ 's as coefficients, i.e. $\delta(X)=\sum_{i=0}^{d} \delta_{i} \cdot X^{i}$. Consider the following polynomial-time relation:

$$
\mathfrak{R}:=\left\{\left(p k, \mathrm{com}^{*}\right) ;(\tilde{m}, \tilde{r}): \operatorname{com}^{*}=\operatorname{Commit}(p k, \tilde{m} ; \tilde{r})\right\} .
$$

${ }^{5}$ For notational convenience, we assume that the product of commitments give commitments to the sum of messages using the sum of the randomness as randomness, à la Pedersen 32 . 
Compute $\tilde{m}=\delta(m)$ and $\tilde{r}=\sum_{i=0}^{d} r_{i} \cdot m^{i}$. Note that both values $\tilde{m}, \tilde{r}$ can be computed efficiently as a function of the signing key $(\boldsymbol{\delta}, \mathbf{r})$ and the message to be signed. Using crs as common reference string, generate a NIZK argument $\pi$ that $\left(p k, \prod_{i=0}^{d}\left(\operatorname{com}_{i}\right)^{m^{i}}\right) \in \mathfrak{L}_{\mathfrak{R}}$, the language generated by the above relation $\mathfrak{R}$. Output $\sigma=\pi$.

Verification. Given a pair $(m, \sigma)$, parse $\sigma$ as $\sigma=\pi$ and compute com $^{*}=$ $\prod_{i=0}^{d}\left(\operatorname{com}_{i}\right)^{m^{i}}$. Output the same as $\operatorname{Ver}\left(\mathrm{crs}, \pi,\left(p k, \mathrm{com}^{*}\right)\right)$.

Let us first argue that the signature scheme satisfies the correctness property. This follows from the fact that $\mathcal{C O} \mathcal{M}$ is linearly homomorphic (cf. Section 2.2):

$$
\operatorname{com}^{*}=\prod_{i=0}^{d}\left(\operatorname{com}_{i}\right)^{m^{i}}=\prod_{i=0}^{d} \operatorname{Commit}\left(\delta_{i} \cdot m^{i} ; r_{i} \cdot m^{i}\right)=\operatorname{Commit}(\underbrace{\sum_{i=0}^{d} \delta_{i} \cdot m^{i}}_{\tilde{m}} ; \underbrace{\sum_{i=0}^{d} r_{i} \cdot m^{i}}_{\tilde{r}}) .
$$

We prove the following result:

Theorem 2. Assume that $\mathcal{C O} \mathcal{M}$ is perfectly hiding and computationally binding, and that $\mathcal{N I Z K}$ is a NIZK argument of knowledge system for relation $\mathfrak{R}$. Then the scheme $\mathcal{S S}$ described above is $(\ell, \gamma)$-one-more unforgeable, as long as

$$
\ell=d \log |\mathbb{F}| \quad \text { and } \quad \gamma=\frac{\log |\mathbb{F}|}{|\sigma|} .
$$

\subsection{Proof of Theorem 2}

To prove the theorem we will rely on the following property of any perfectly hiding commitment scheme $\mathcal{C O} \mathcal{M}=$ (Setup, Commit). Define the following experiment $\operatorname{Exp}_{\mathcal{C O M}, \mathrm{A}}^{\text {guess }}(\kappa, \ell, d)$, featuring an unbounded adversary $\mathrm{A}$ :

1. Run $p k \leftarrow \operatorname{Setup}\left(1^{\kappa}\right)$ and sample $x_{1}, \ldots, x_{d} \in \mathcal{M}$ uniformly at random. Compute $\operatorname{com}_{i}=\operatorname{Commit}\left(p k, x_{i} ; r_{i}\right)$ and give $\left(\left\{\operatorname{com}_{i}\right\}_{i=1}^{d}, p k\right)$ to A. Store $s=\left(\left\{x_{i}\right\}_{i=1}^{d},\left\{r_{i}\right\}_{i=1}^{d}\right)$.

2. The adversary can access adaptively oracle $\mathcal{O}^{\ell}(s, \cdot)$. Let $\Lambda \in\{0,1\}^{\lambda}$ be the overall information retrieved by $\mathrm{A}$ (with $\lambda \leq \ell$ ).

3. The adversary can open a subset of size $t$ of $\left(x_{1}, \ldots, x_{d}\right)$ : Given a set of indexes $\left(i_{1}, \ldots, i_{t}\right)$ such that each $i_{j} \in[d]$, the values $\left(\left\{x_{i_{j}}\right\}_{j=1}^{t},\left\{r_{i_{j}}\right\}_{j=1}^{t}\right)$ are forwarded to $A$.

4. The experiment returns 1 if $\mathrm{A}$ outputs the remaining values $x_{i}$, for all $i \in$ $[d] \backslash\left\{i_{1}, \ldots, i_{t}\right\}$.

Lemma 2. Let $\mathcal{C O} \mathcal{M}=$ (Setup, Commit) be a perfectly hiding commitment scheme with message space $\mathcal{M}$. Then for every computationally unbounded adversary $\mathrm{A}$ we have that

$$
\mathbb{P}\left[\operatorname{Exp}_{\mathcal{C O M}, \mathrm{A}}^{\text {guess }}(\kappa, \ell, d)=1\right] \leq \frac{2^{\lambda}}{|\mathcal{M}|^{d-t}}
$$


The proof of Lemma 2 appears in the full version of this paper.

We now prove Theorem 2. Let A be a PPT machine running in experiment $\operatorname{Exp}_{\mathcal{S} S, A}^{\text {one-more }}(\kappa, \ell, \gamma)$. We recall how the experiment is held for our scheme $\mathcal{S} \mathcal{S}$.

1. The signing key $s k=(\boldsymbol{\delta}, \mathbf{r})$ and the verification key $v k=\left(p k,\left\{c o m_{i}\right\}_{i=0}^{d}\right)$ are computed. In particular, $p k \leftarrow \operatorname{Setup}\left(1^{\kappa}\right)$ and crs $\leftarrow \operatorname{Init}\left(1^{\kappa}\right)$. Here, $\boldsymbol{\delta}=$ $\left(\delta_{0}, \ldots, \delta_{d}\right) \leftarrow \mathbb{F}^{d+1}, \operatorname{com}_{i}=\operatorname{Commit}\left(\delta_{i} ; r_{i}\right)$ and $\mathbf{r}=\left(r_{0}, \ldots, r_{d}\right) \leftarrow \mathbb{F}^{d+1}$.

2. The adversary $\mathrm{A}$ is given $v k$ and can access oracles $\operatorname{Sign}(s k, \cdot)$ and $\mathcal{O}^{\ell}(s k, \cdot)$.

- The signing oracle $\operatorname{Sign}(s k, m)$ computes $\tilde{m}=\sum_{i=0}^{d} \delta_{i} \cdot m^{i}$ and $\tilde{r}=$ $\sum_{i=0}^{d} r_{i} \cdot m^{i}$, together with an argument $\pi$ that $\left(p k, c o m^{*}\right) \in \mathcal{L}_{\mathfrak{R}}$ for $\operatorname{com}^{*}=\operatorname{Commit}(\tilde{m} ; \tilde{r})$; hence, it returns $\sigma=\pi$.

- The leakage oracle $\mathcal{O}^{\ell}(s k, f)$ returns $f(s k)$.

3. A outputs $n$ pairs $\left(m_{1}, \pi_{1}\right), \ldots,\left(m_{n}, \pi_{n}\right)$.

4. The experiment outputs 1 iff the following conditions are satisfied:

(a) $\operatorname{Verify}\left(v k,\left(m_{i}, \sigma_{i}\right)\right)=1$ and $m_{i} \notin \mathcal{Q}$, for all $i \in[n]$.

(b) The messages $m_{1}, \ldots, m_{n}$ are pairwise distinct.

(c) $n \geq\lfloor\lambda /(\gamma|\sigma|)\rfloor+1$.

The proof proceeds by a series of games.

Game $_{0}$. This is the real experiment, as described above.

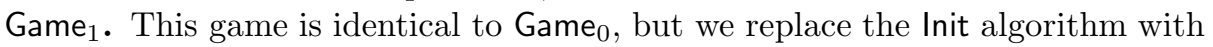
$(\mathrm{crs}, t k) \leftarrow \operatorname{Sim}_{1}\left(1^{\kappa}\right)$. Moreover, each time a signing query for message $m$ is asked, we simulate the argument by running $\pi \leftarrow \operatorname{Sim}_{2}\left(t k,\left(p k, \operatorname{com}^{*}\right)\right)$. Everything else remains the same.

By a standard argument, the multi-theorem zero-knowledge property of the argument system implies that $\mathbb{P}\left[\mathrm{A}\right.$ wins Game $\left._{0}\right]$ is negligibly close to $\mathbb{P}[$ A wins Game 1 .

Game $_{2}$. This game is identical to $\mathrm{Game}_{1}$, but the common reference string is sampled as $(\mathrm{crs}, t k, x k) \leftarrow \mathrm{Xtr}_{1}\left(1^{\kappa}\right)$ and before outputting 1 we check that all arguments contained in A's forgeries can be extracted via $\mathrm{Xtr}_{2}(x k, \cdot)$.

By a standard argument, (true) simulation extractability of $\mathcal{N} \mathcal{I} \mathcal{Z} \mathcal{K}$ implies that $\mathbb{P}\left[\mathrm{A}\right.$ wins Game $\left._{1}\right]$ is negligibly close to $\mathbb{P}\left[\mathrm{A}\right.$ wins Game $\left._{2}\right]$.

Now, we show that $\mathbb{P}\left[\mathrm{A}\right.$ wins $\left.\mathrm{Game}_{2}\right]$ is negligible which proves the theorem. Define the following event $\mathrm{Bad}$ in $\mathrm{Game}_{2}$ : The event occurs whenever for at least one of the forgeries $\left(m_{j}, \sigma_{j}\right)$ returned by $\mathrm{A}$ it holds that $\tilde{m}_{j}^{\prime} \neq \sum_{i=0}^{d} \delta_{i} \cdot m_{j}^{i}$ for $\left(\tilde{m}_{j}^{\prime}, \tilde{r}_{j}^{\prime}\right) \leftarrow \operatorname{Xtr}_{2}\left(x k, \pi_{j}\right)$. In other words, there exists a valid pair $\left(m_{j}, \sigma_{j}\right)$ for which the extracted value $\tilde{m}_{j}^{\prime}$ is not the evaluation of $m_{j}$ through the polynomial $\delta(X)$ having $\boldsymbol{\delta}$ as coefficients. We write

$$
\begin{aligned}
& \mathbb{P}[\text { A wins Game } 2] \leq \mathbb{P}[\text { A wins Game } 2 \wedge \text { Bad }]+\mathbb{P}[\text { A wins Game } 2 \wedge \overline{B a d}] \\
& \leq \operatorname{negl}(\kappa) \text {, }
\end{aligned}
$$

where the last inequality comes from the two claims below.

Claim. $\mathbb{P}\left[\right.$ A wins Game $\left.{ }_{2} \wedge \overline{B a d}\right] \leq 2^{\lambda} /|\mathbb{F}|^{n}$. 
Proof. By contradiction, assume that $\mathbb{P}\left[\mathrm{A}\right.$ wins Game Ga $\left._{2} \wedge \overline{B a d}\right]>2^{\lambda} /|\mathbb{F}|^{n}$. We build a PPT reduction B (running A) which wins the game of experiment $\operatorname{Exp}_{\mathcal{C O M}, \mathrm{B}}^{\text {guess }}(\kappa, \ell, d+1)$ with at least the same advantage.

1. Given $\left\{\operatorname{com}_{i}=\operatorname{Commit}\left(\delta_{i} ; r_{i}\right)\right\}_{i=0}^{d}$ as input, implicitly define $s k:=(\boldsymbol{\delta}, \mathbf{r})$, where

$$
\boldsymbol{\delta}=\left(\delta_{0}, \delta_{1}, \ldots, \delta_{d}\right) \quad \mathbf{r}=\left(r_{0}, r_{1}, \ldots, r_{d}\right) .
$$

Run $(\mathrm{crs}, t k, x k) \leftarrow \mathrm{Xtr}_{1}\left(1^{\kappa}\right)$ and give $v k:=\left(\mathrm{crs}, p k,\left\{\operatorname{com}_{i}\right\}_{i=0}^{d}\right)$ to $\mathrm{A}$.

2. Whenever $A$ asks a leakage query $f$ to $\mathcal{O}^{\ell}((\boldsymbol{\delta}, \mathbf{r}), \cdot)$, forward the same query to $\mathcal{O}^{\ell}(s, \cdot)$ (where $s=(\boldsymbol{\delta}, \mathbf{r})$ ). Give to A the same value returned by $\mathcal{O}^{\ell}(s)$.

3. Whenever A asks a signing query $m$ to $\operatorname{Sign}(s k, \cdot)$, answer as follows. Simulate an argument $\pi \leftarrow \operatorname{Sim}\left(t k,\left(p k, c^{*} m^{*}\right)\right)$ where $\operatorname{com}^{*}=\prod_{i=0}^{d}\left(\operatorname{com}_{i}\right)^{m^{i}}$. Give $\sigma=\pi$ to $\mathrm{A}$.

4. Let $\left(m_{1}, \sigma_{1}\right), \ldots,\left(m_{n}, \sigma_{n}\right)$ be the forgeries output by A. Ask to open the last $t:=d+1-n$ values, i.e. $\left(\left\{\delta_{i}\right\}_{i=n}^{d},\left\{r_{i}\right\}_{i=n}^{d}\right)$. Set $\tilde{\delta}_{i}=\delta_{i}$ for each $i=n, \ldots, d$.

5. For each of the values $\sigma_{i}=\pi_{i}$ returned by A compute $\left(\tilde{m}_{i}^{\prime}, \tilde{r}_{i}^{\prime}\right) \leftarrow \operatorname{Xtr}_{2}\left(x k, \pi_{i}\right)$.

Solve the following linear system:

$$
\left(\begin{array}{ccc}
1 m_{1} & \ldots & m_{1}^{n-1} \\
& \ddots & \\
1 m_{n} & \ldots & m_{n}^{n-1}
\end{array}\right) \cdot\left(\begin{array}{c}
\delta_{0} \\
\vdots \\
\delta_{n-1}
\end{array}\right)=\left(\begin{array}{c}
y_{0} \\
\vdots \\
y_{n-1}
\end{array}\right)
$$

where each of the values $y_{i}$ is computed from known values as $y_{i}=\tilde{m}_{i}^{\prime}-$ $\sum_{j=n}^{d} \tilde{\delta}_{j} \cdot m_{i}^{j}$.

6. Output $\left(\delta_{1}, \ldots, \delta_{n}\right)$.

Note that B perfectly simulates the environment for A in Game 2 . The choice of the parameters $\gamma=\log |\mathbb{F}| /|\sigma|$ and $\ell=d \log |\mathbb{F}|$ (as in the theorem statement), ensures that $1 \leq n \leq d+1$. In particular, the total number of field elements known by $\mathrm{B}$ is at most $\frac{\lambda}{\log |\mathbb{F}|}+\left(d-\left\lfloor\frac{\lambda}{\gamma|\sigma|}\right\rfloor\right)<d+1$, and thus there is some entropy left in the commitments.

Moreover, as the values $\left(m_{1}, \ldots, m_{n}\right)$ are pairwise distinct, the matrix of Eq. (11) has full rank and the linear system always admits a solution. Since the event $B a d$ does not happen, we have that $\tilde{m}_{i}^{\prime}=\tilde{m}_{i}=\delta\left(m_{i}\right)$ (for all $i \in[n]$ ), and thus the solution $\left(\delta_{1}, \ldots, \delta_{n}\right)$ corresponds to the same elements in the vector $\boldsymbol{\delta}$. The above contradicts Lemma 2, as

$$
\mathbb{P}\left[\operatorname{Exp}_{\mathcal{C O M}, \mathrm{B}}^{\text {guess }}(\kappa, \ell, d+1)=1\right] \geq \mathbb{P}[\mathrm{A} \text { wins Game } 2 \wedge \overline{B a d}]>\frac{2^{\lambda}}{|\mathbb{F}|^{n}}
$$

Claim. $\mathbb{P}[\mathrm{A}$ wins Game $2 \wedge \operatorname{Bad}] \leq \operatorname{negl}(\kappa)$.

Proof. Assume that $\mathbb{P}\left[\mathrm{A}\right.$ wins Game $\mathrm{G}_{2} \wedge$ Bad $]>1 /$ poly $(\kappa)$ for infinitely many $\kappa$. We build an attacker $C$ breaking the binding property of the commitment scheme $\mathcal{C O} \mathcal{M}$ with non-negligible advantage. A description of $\mathrm{C}$ follows: 
1. Receive the public parameter $p k$ for $\mathcal{C O} \mathcal{M}$. Choose $\delta_{0}, \ldots, \delta_{d} \leftarrow \mathbb{F}$ and compute commitments $\operatorname{com}_{i}=\operatorname{Commit}\left(p k, \delta_{i} ; r_{i}\right)$ for randomly chosen $r_{0}$, $\ldots, r_{d} \leftarrow \mathbb{F}$. Generate $(\mathrm{crs}, t k, x k) \leftarrow \mathrm{Xtr}_{1}\left(1^{\kappa}\right)$.

2. Run $\mathrm{A}$ with input $v k:=\left(\mathrm{crs}, p k,\left\{\operatorname{com}_{i}\right\}_{i=0}^{d}\right)$ and answer signing/leakage queries from $A$ as it would be done in $\mathrm{Game}_{2}$.

3. When A outputs $\left(m_{1}, \pi_{1}\right), \ldots,\left(m_{n}, \pi_{n}\right)$, extract the witness $\left(\tilde{m}_{i}^{\prime}, \tilde{r}_{i}^{\prime}\right)$ from each argument of knowledge $\pi_{i}$, for $i \in[n]$.

4. If there exists an index $j \in[n]$ such that $\tilde{m}_{j}^{\prime} \neq \delta\left(m_{j}\right)$, compute $\operatorname{com}_{j}^{*}=$ $\prod_{i=0}^{d}\left(\operatorname{com}_{i}\right)^{m_{j}^{i}}, \tilde{m}_{j}=\sum_{i=0}^{d} \delta_{i} \cdot m_{j}^{i}$ and $\tilde{r}_{j}=\sum_{i=0}^{d} r_{i} \cdot m_{j}^{i}$ and output $\left(\operatorname{com}_{j}^{*},\left(\tilde{m}_{j}, \tilde{r}_{j}\right),\left(\tilde{m}_{j}^{\prime}, \tilde{r}_{j}^{\prime}\right)\right)$; otherwise abort and output $\perp$.

Notice that if Game 2 outputs 1 and event Bad occurs, then C outputs a valid pair breaking the binding property of $\mathcal{C O} \mathcal{M}$ with non-negligible probability (a contradiction). This is because both $\left(\tilde{m}_{j}, \tilde{r}_{j}\right)$ and $\left(\tilde{m}_{j}^{\prime}, \tilde{r}_{j}^{\prime}\right)$ are valid openings for $\operatorname{com}_{j}^{*}$ and moreover Bad implies that $\left(\tilde{m}_{j}, \tilde{r}_{j}\right) \neq\left(\tilde{m}_{j}^{\prime}, \tilde{r}_{j}^{\prime}\right)$.

\subsection{A Concrete Instantiation}

In this section we show how to instantiate our signature scheme, reducing security to the DLIN assumption [5]. For each of the building blocks we present an instantiation and concrete parameters.

In the following let $\mathbb{G}$ be a cyclic group of order a prime number $q$. Before introducing our concrete construction, let us recall the DLIN assumption:

Definition 4. The DLIN assumption states that for any PPT algorithm A it holds that

$$
\mid \mathbb{P}\left[\mathrm{A}\left(\mathbb{G},\left(g, g_{1}, g_{2}, g_{1}^{a}, g_{2}^{b}, g^{c}\right)\right)=1\right]-\mathbb{P}\left[\mathrm{A}\left(\mathbb{G},\left(g, g_{1}, g_{2}, g_{1}^{a}, g_{2}^{b}, g^{a+b}\right)=1\right] \mid \leq \operatorname{negl}(\kappa),\right.
$$

where $g, g_{1}, g_{2} \leftarrow \mathbb{G}$ and $a, b, c \leftarrow \mathbb{F}_{q}$.

$\mathcal{C O M}$ : We use Pedersen commitments. The setup algorithm Setup outputs public parameters $p k=\left(h_{1}, h_{2}\right)$, where $h_{1}$ is a generator for $\mathbb{G}$ and $h_{2}=$ $h_{1}^{a}$ for a random $a \in \mathbb{F}_{q}$. The commitment to an element $m \in \mathbb{F}_{q}$ using randomness $r \leftarrow \mathbb{F}_{q}$ is computed as com $=\operatorname{Commit}(p k, m ; r):=h_{1}^{m} \cdot h_{2}^{r}$. Whenever we want to open the commitment, we reveal $(m, r)$.

Note that Pedersen commitment is linearly homomorphic: given $\operatorname{com}_{1}=$ $\operatorname{Commit}\left(m_{1} ; r_{1}\right)$ and $\operatorname{com}_{2}=\operatorname{Commit}\left(m_{2} ; r_{2}\right)$ it holds that

$$
\operatorname{com}_{1} \cdot \operatorname{com}_{2}=h_{1}^{m_{1}+m_{2}} \cdot h_{2}^{r_{1}+r_{2}}=\operatorname{Commit}\left(m_{1}+m_{2} ; r_{1}+r_{2}\right) .
$$

Moreover, for all constants $c \in \mathbb{F}_{q}$ we have that $\operatorname{com}^{c}=h_{1}^{c \cdot m} \cdot h_{2}^{c \cdot r}=$ Commit $(c \cdot m ; c \cdot r)$.

$\mathcal{N I Z K}$ : Recall that our relation is as follows:

$$
\Re=\left\{\left(p k, \operatorname{com}^{*}\right) ;(\tilde{m}, \tilde{r}): \operatorname{com}^{*}=\operatorname{Commit}(p k, \tilde{m} ; \tilde{r})\right\} .
$$


When using Pedersen commitment, we get

$$
\operatorname{com}^{*}=\prod_{i=0}^{d}\left(\operatorname{com}_{i}\right)^{m^{i}}=\prod_{i=0}^{d}\left(h_{1}^{\delta_{i}+a r_{i}}\right)^{m^{i}}=h_{1}^{\sum_{i=0}^{d} \delta_{i} m^{i}+a r_{i} m^{i}}=h_{1}^{\tilde{m}+a \cdot \tilde{r}} .
$$

Thus, we can reduce the proof of knowledge of an opening for $\mathrm{com}^{*}$ to the proof of knowledge of a discrete logarithm. Groth [18 gives a simulationextractable NIZK for proving knowledge of discrete logarithms of a group element. We remark that the length of a proof is constant, and in particular independent of the degree $d$ of the polynomial.

Alternatively, as true simulation-extractability is sufficient for our construction, one could instantiate the NIZK using the transformation of [10], which requires a standard (non-simulation-extractable) NIZK and a labeled CCA-secure encryption scheme.

\section{Application to Leaky Identification}

We show how to apply one-more unforgeability to the context of (leaky) identification protocols. In a public key identification scheme a prover with public key $v k$ attempts to prove its identity to a verifier holding $v k$. More formally, an identification scheme $\mathcal{I D}=($ PGen, KGen, P, V) consists of four PPT algorithms described as follows: (1) The parameters generation algorithm takes as input the security parameter and outputs public parameters params $\leftarrow$ PGen $\left(1^{\kappa}\right)$, shared by all users 6 (2) The key generation algorithm takes as input the security parameter and outputs a verification key/secret key pair $(v k, s k) \leftarrow \mathrm{KGen}\left(1^{\kappa}\right)$. (3) $\mathrm{P}$ and $\mathrm{V}$ are probabilistic Turing machines interacting in a protocol $(\mathrm{P}(s k) \rightleftarrows$ $\mathrm{V})(v k)$; at the end of the execution $\mathrm{V}$ outputs a judgment $d \in\{0,1\}$, where $d=1$ means that the identification was successful.

Following [1], we define a leaky variant of the standard notion of active security (dubbed active $\ell$-security under pre-impersonation attacks with leakage), where an adversary, in a first stage, is given black-box access to the honest prover, and in a second stage is given one shot to convince the verifier. In the leaky case, during the first phase, the adversary can also access adaptively a leakage oracle $\mathcal{O}^{\ell}(s k)$.

We then show that the below standard way (see 2]) of constructing an identification scheme $\mathcal{I D}$ from a signature scheme $\mathcal{S} \mathcal{S}=\left(\mathrm{KGen}^{\prime}\right.$, Sign, Verify), achieves active $\ell$-security under pre-impersonation attacks with leakage provided that $\mathcal{S} \mathcal{S}$ is one-more unforgeable.

- Parameters generation. Algorithm PGen samples the public parameters params for the signature schemes (if any).

- Key Generation. Algorithm KGen runs the key generation algorithm of the signature scheme, obtaining $(v k, s k) \leftarrow \mathrm{KGen}^{\prime}\left(1^{\kappa}\right)$.

- Identification protocol. The interaction is as follows: (a) The verifier sends a random $m^{*} \leftarrow \mathcal{M}$ to the prover; (b) The prover replies with $\sigma^{*} \leftarrow$ $\operatorname{Sign}\left(s k, m^{*}\right)$; (c) The verifier outputs Verify $\left(v k,\left(m^{*}, \sigma^{*}\right)\right)$.

${ }^{6}$ In what follows all algorithms take as input params, but we omit to explicitly write this for ease of notation. 
Theorem 3. Assume that $\mathcal{S S}$ is $(\ell, \gamma)$-constrained-one-more unforgeable. Then $\mathcal{I D}$ from above is actively $\ell$-secure under pre-impersonation attacks with leakage.

For space reasons, a formal definition and a proof of the above theorem are deferred to the full version of this paper.

\section{References}

1. Alwen, J., Dodis, Y., Wichs, D.: Leakage-resilient public-key cryptography in the bounded-retrieval model. In: Halevi, S. (ed.) CRYPTO 2009. LNCS, vol. 5677, pp. 36-54. Springer, Heidelberg (2009)

2. Bellare, M., Fischlin, M., Goldwasser, S., Micali, S.: Identification protocols secure against reset attacks. In: Pfitzmann, B. (ed.) EUROCRYPT 2001. LNCS, vol. 2045, pp. 495-511. Springer, Heidelberg (2001)

3. Bellare, M., Rogaway, P.: Random oracles are practical: A paradigm for designing efficient protocols. In: ACM Conference on Computer and Communications Security, pp. 62-73 (1993)

4. Bitansky, N., Canetti, R., Halevi, S.: Leakage-tolerant interactive protocols. In: Cramer, R. (ed.) TCC 2012. LNCS, vol. 7194, pp. 266-284. Springer, Heidelberg (2012)

5. Boneh, D., Boyen, X., Shacham, H.: Short Group Signatures. In: Franklin, M. (ed.) CRYPTO 2004. LNCS, vol. 3152, pp. 41-55. Springer, Heidelberg (2004)

6. Boyle, E., Segev, G., Wichs, D.: Fully leakage-resilient signatures. In: Paterson, K.G. (ed.) EUROCRYPT 2011. LNCS, vol. 6632, pp. 89-108. Springer, Heidelberg (2011)

7. Brakerski, Z., Kalai, Y.T., Katz, J., Vaikuntanathan, V.: Overcoming the hole in the bucket: Public-key cryptography resilient to continual memory leakage. In: FOCS, pp. 501-510 (2010)

8. Davì, F., Dziembowski, S., Venturi, D.: Leakage-resilient storage. In: Garay, J.A., De Prisco, R. (eds.) SCN 2010. LNCS, vol. 6280, pp. 121-137. Springer, Heidelberg (2010)

9. Dodis, Y., Haralambiev, K., López-Alt, A., Wichs, D.: Cryptography against continuous memory attacks. In: FOCS, pp. 511-520 (2010)

10. Dodis, Y., Haralambiev, K., López-Alt, A., Wichs, D.: Efficient public-key cryptography in the presence of key leakage. In: Abe, M. (ed.) ASIACRYPT 2010. LNCS, vol. 6477, pp. 613-631. Springer, Heidelberg (2010)

11. Dodis, Y., Lewko, A.B., Waters, B., Wichs, D.: Storing secrets on continually leaky devices. In: FOCS, pp. 688-697 (2011)

12. Dodis, Y., Ostrovsky, R., Reyzin, L., Smith, A.: Fuzzy extractors: How to generate strong keys from biometrics and other noisy data. SIAM J. Comput. 38(1), 97-139 (2008)

13. Dziembowski, S., Pietrzak, K.: Leakage-resilient cryptography. In: FOCS, pp. 293-302 (2008)

14. Faust, S., Kiltz, E., Pietrzak, K., Rothblum, G.N.: Leakage-resilient signatures. In: Micciancio, D. (ed.) TCC 2010. LNCS, vol. 5978, pp. 343-360. Springer, Heidelberg (2010)

15. Faust, S., Kohlweiss, M., Marson, G.A., Venturi, D.: On the non-malleability of the Fiat-Shamir transform. In: Galbraith, S., Nandi, M. (eds.) INDOCRYPT 2012. LNCS, vol. 7668, pp. 60-79. Springer, Heidelberg (2012)

16. Fiat, A., Shamir, A.: How to prove yourself: Practical solutions to identification and signature problems. In: Odlyzko, A.M. (ed.) CRYPTO 1986. LNCS, vol. 263, pp. 186-194. Springer, Heidelberg (1987) 
17. Garg, S., Jain, A., Sahai, A.: Leakage-resilient zero knowledge. In: Rogaway, P. (ed.) CRYPTO 2011. LNCS, vol. 6841, pp. 297-315. Springer, Heidelberg (2011)

18. Groth, J.: Simulation-sound NIZK proofs for a practical language and constant size group signatures. In: Lai, X., Chen, K. (eds.) ASIACRYPT 2006. LNCS, vol. 4284, pp. 444-459. Springer, Heidelberg (2006)

19. Groth, J., Ostrovsky, R., Sahai, A.: Non-interactive zaps and new techniques for NIZK. In: Dwork, C. (ed.) CRYPTO 2006. LNCS, vol. 4117, pp. 97-111. Springer, Heidelberg (2006)

20. Groth, J., Ostrovsky, R., Sahai, A.: New techniques for noninteractive zeroknowledge. J. ACM 59(3), 11 (2012)

21. Halevi, S., Lin, H.: After-the-fact leakage in public-key encryption. In: Ishai, Y. (ed.) TCC 2011. LNCS, vol. 6597, pp. 107-124. Springer, Heidelberg (2011)

22. Hofheinz, D., Jager, T., Kiltz, E.: Short signatures from weaker assumptions. In: Lee, D.H., Wang, X. (eds.) ASIACRYPT 2011. LNCS, vol. 7073, pp. 647-666. Springer, Heidelberg (2011)

23. Juels, A., Weis, S.A.: Authenticating pervasive devices with human protocols. In: Shoup, V. (ed.) CRYPTO 2005. LNCS, vol. 3621, pp. 293-308. Springer, Heidelberg (2005)

24. Katz, J., Vaikuntanathan, V.: Signature schemes with bounded leakage resilience. In: Matsui, M. (ed.) ASIACRYPT 2009. LNCS, vol. 5912, pp. 703-720. Springer, Heidelberg (2009)

25. Kiltz, E., Pietrzak, K., Cash, D., Jain, A., Venturi, D.: Efficient authentication from hard learning problems. In: Paterson, K.G. (ed.) EUROCRYPT 2011. LNCS, vol. 6632, pp. 7-26. Springer, Heidelberg (2011)

26. Kiltz, E., Pietrzak, K., Szegedy, M.: Digital signatures with minimal overhead from indifferentiable random invertible functions. In: Canetti, R., Garay, J.A. (eds.) CRYPTO 2013, Part I. LNCS, vol. 8042, pp. 571-588. Springer, Heidelberg (2013)

27. Kocher, P.C.: Timing attacks on implementations of diffie-hellman, RSA, DSS, and other systems. In: Koblitz, N. (ed.) CRYPTO 1996. LNCS, vol. 1109, pp. 104-113. Springer, Heidelberg (1996)

28. Kocher, P.C., Jaffe, J., Jun, B.: Differential power analysis. In: Wiener, M. (ed.) CRYPTO 1999. LNCS, vol. 1666, pp. 388-397. Springer, Heidelberg (1999)

29. Malkin, T., Teranishi, I., Vahlis, Y., Yung, M.: Signatures resilient to continual leakage on memory and computation. In: Ishai, Y. (ed.) TCC 2011. LNCS, vol. 6597, pp. 89-106. Springer, Heidelberg (2011)

30. Naor, M., Segev, G.: Public-key cryptosystems resilient to key leakage. IACR Cryptology ePrint Archive, 105 (2009)

31. Nielsen, J.B., Venturi, D., Zottarel, A.: On the connection between leakage tolerance and adaptive security. In: Kurosawa, K., Hanaoka, G. (eds.) PKC 2013. LNCS, vol. 7778, pp. 497-515. Springer, Heidelberg (2013)

32. Pedersen, T.P.: Non-interactive and information-theoretic secure verifiable secret sharing. In: Feigenbaum, J. (ed.) CRYPTO 1991. LNCS, vol. 576, pp. 129-140. Springer, Heidelberg (1992)

33. Pietrzak, K.: A leakage-resilient mode of operation. In: Joux, A. (ed.) EUROCRYPT 2009. LNCS, vol. 5479, pp. 462-482. Springer, Heidelberg (2009)

34. Pointcheval, D., Stern, J.: Security arguments for digital signatures and blind signatures. J. Cryptology 13(3), 361-396 (2000)

35. Quisquater, J.-J., Samyde, D.: ElectroMagnetic analysis (EMA): Measures and counter-measures for smart cards. In: Attali, S., Jensen, T. (eds.) E-smart 2001. LNCS, vol. 2140, pp. 200-210. Springer, Heidelberg (2001)

36. De Santis, A., Di Crescenzo, G., Ostrovsky, R., Persiano, G., Sahai, A.: Robust noninteractive zero knowledge. In: Kilian, J. (ed.) CRYPTO 2001. LNCS, vol. 2139, pp. 566-598. Springer, Heidelberg (2001) 